\title{
Prognostic Significance of Preoperative Serum Uric Acid to Lymphocyte Ratio in Advanced Gastric Cancer A Propensity Score-Matched Analysis
}

\section{Li Heng Liu}

The First Affiliated Hospital of Chongqing Medical University

Shan Shan Hu

The First Affiliated Hospital of Chongqing Medical University

Cheng Lei Liao

The First Affiliated Hospital of Chongqing Medical University

ziwei wang ( $\square$ sirius979xx3@sina.com )

The First Affiliated Hospital of Chongqing Medical University

\section{Research}

Keywords: Gastric cancer, serum uric acid, lymphocyte, prognosis

Posted Date: February 17th, 2021

DOl: https://doi.org/10.21203/rs.3.rs-213684/v1

License: (c) (i) This work is licensed under a Creative Commons Attribution 4.0 International License. Read Full License 


\section{Abstract}

Purpose: Gastric cancer ranks sixth and second in incidence and mortality among all cancer. The purpose of our research was to evaluate the prognostic value of uric acid to lymphocyte ratio (ULR) for advanced gastric cancer (AGC) patients after gastrectomy with D2 lymphadenectomy.

Methods: In this research, we included 287 AGC patients underwent gastrectomy with D2 lymphadenectomy. These patients were followed up for 5 years, and their clinicopathological data were collected. All patients were divided into two groups based on the preoperative ULR level. Then we established the propensity score matching (PSM) cohort to confirm our results. Finally, The clinical characteristics and survival indexes of the two groups in the PSM cohort and the entire cohort were compared.

Results $\llbracket W e$ found that age and gender were significantly correlated with the ULR level. Multivariate analysis for the entire cohort and PSM cohort showed that high levels of ULR, poor differentiation, postoperative pathology of $\mathrm{T}_{4}$ stage, and regional lymph node metastasis were independent prediction factors for poor outcomes of overall survival (OS) and Disease-free survival (DFS) in patients with AGC after gastrectomy with D2 lymphadenectomy. Survival analysis showed that the OS and DFS in the high level ULR group were significantly shorter than in the low level ULR group (log-rank $P<0.001$ ).

Conclusion: High level ULR is a poor predictive factor for patients with AGC underwent gastrectomy with D2 lymphadenectomy, and high levels of ULR predict shorter OS and DFS.

\section{Introduction}

Gastric cancer is one of the most common cancers globally, and the mortality rate ranks among the top three of all cancers ${ }^{[1]}$. Despite the advancements in surgical methods, preoperative neoadjuvant chemotherapy, postoperative radiotherapy and chemotherapy, and immunotherapy, the 5-year survival rate of patients with advanced gastric cancer (AGC) is still poor and results in the untimely diagnosis. Therefore, we urgently need a simple and effective biomarker to evaluate the postoperative prognosis of gastric cancer and help surgeons make a suitable treatment plan.

The traditional judgment of tumor prognosis is based mainly on a series of pathological factors, including tumor differentiation, scope of invasion, and number of lymph node metastasis. With advancing research, more and more tumor prognostic markers have been discovered, among which a variety of peripheral serum biomarkers are particularly important for predicting the prognosis of tumors. Serological biomarkers are easy and cheap to obtain, so people are paying more attention to them. Examples of serological biomarkers are neutrophil to lymphocyte ratio, C-reactive protein, and interleukin6 . All of the above indexes had been widely used to predict the prognosis of gastric cancer ${ }^{[2][3][4]}$.

Uric acid (UA) consists of purine nucleotides. Hyperuricemia is related to the occurrence and development of many diseases, and can lead to obesity, type 2 diabetes (T2DM), insulin resistance, 
hypertension, gout, and other diseases. Hyperuricemia can also increase the risk of mortality from cancer $^{[5]}$. In recent years, various studies have reported that serum uric acid (SUA) is correlated with the incidence and progression of AGC, esophageal cancer, colon cancer, pancreatic cancer, and other tumors $^{[6][7][8][9]}$.

Peripheral blood lymphocytes are composed mainly of T cells, B cells, and a small number of natural killer cell. It is generally believed that the systemic inflammatory response could be activated during carcinogenesis along with a significant decrease in lymphocytes, which leads to a weakening of immune function with resultant tumor growth, invasion, and metastasis. At present, there are a variety of lymphocyte-based prognostic assessment tools, all of which are helpful for judging the prognosis of cancer patients after treatment ${ }^{[10][11]}$. It was first reported by Wei in 2018 that serum uric acid to lymphocyte ratio (ULR) could predict the prognosis of patients with rheumatic heart disease underwent valve replacement ${ }^{[12]}$. Recently, it was confirmed that ULR can independently predict long-term survival in postoperative patients with lung cancer, and its predictive efficiency is higher than serum uric acid and blood lymphocytes alone ${ }^{[13]}$. However, the relationship between ULR and advanced gastric cancer is not clear. For this reason, we explored the significance of ULR in gastric cancer.

\section{Materials And Methods}

\subsection{Subjects}

This was a single center retrospective study. The study was approved by the Ethics Committee of Chongqing Medical University(ID:2020-432).

\subsection{Inclusion and exclusion criteria}

Appropritate patients were included in accordance with following eligibility criteria.

1. Patients were diagnosed gastric adenocarcinoma and tumor invades deeply than the submucosa though postoperative pathology.

2. Patients received gastrectomy with D2 lymphadenectomy.

3. Patients had complete clinical and follow-up data.

4. Patients had received preoperative therapy, such as neoadjuvant radiotherapy and chemotherapy were excluded.

5. No previous malignant tumors or patients had multiple primary tumors history.

6. Patients who had diseases affecting serum uric acid and blood lymphocyte count, such as gout, hematological diseases, kidney diseases, and drug poisoning were excluded.

\subsection{Data Collection and Definitions}


The clinical data of 397 AGC patients were collected from the first affiliated Hospital of Chongqing Medical University (Chongqing, China) during January 2013 to January 2015. The data included age, sex, body mass index (BMI), tumor differentiation, $T$ stage, number of lymph node metastasis (LNM), and hypertension and type 2 diabetes comorbidity. All factors were consistent with the definition of AJCC $8^{\text {th }}$ edition.

All serological indexes were examined at least 3 days before surgery. Serum uric acid, serum albumin, and lymphocyte counts came from the results of routine blood and biochemical tests in our hospital. According to the clinical standard of our hospital, albumin ( $>4 \mathrm{~g} / \mathrm{dL}$ ), serum uric acid (male 208-428 $\mathrm{mmol} / \mathrm{L}$, female $155-357 \mathrm{mmol} / \mathrm{L})$, and the absolute value of lymphocytes $(1.10-3.2 \mathrm{mmol} / \mathrm{L})$ were defined as normal values.

We followed up every 3 months during the first year, every 6 months during the subsequent 2 years, and once a year after 3 years. The follow-up items included a physical examination, laboratory test, gastroscopy, ultrasound, and chest and abdominal CT examination. If the patient could not come to the clinic, follow-up was done by telephone. Our primary outcome of interest were overall survival (OS), We defined the OS as the length of time during operation day to death from any cause or the end of follow up. Our secondary outcome of interest were disease-free survival(DFS), the DFS was defined as the length of time during operation day to the detection of cancer recurrence or metastasis or death by other cause. The last follow-up visit was in January 2020.

\subsection{Statistical Methods}

We used the "survivalROC" software package of R software to draw the time-dependent ROC curve. The largest Youden's index was selected as the cut-off point. We used Fisher's exact test to compare classified data. Kaplan-Meier and log-rank tests were applied to compare the survival between the two groups. Univariate and multivariate analysis were calculated to predict the prognostic factors for the AGC patients.

To reduce the impact of confounding factors, we formulated a propensity score matching (PSM) model by the nearest neighbor method with a 0.2 caliper width and 1:1 ratio propensity score matching. In the PSM model, the components of the ULR could not participate in the matching $\left[{ }^{14]}\right.$, which indicated that the serum markers from routine blood and biochemical tests were not taken into account in the PS balance.

We used the R software 3.63 (https://www.r-project.org/)) to accomplish all statistical analyses. A p-value $<0.05$ was regarded as statistically significant.

\section{Results}

\subsection{Clinicopathologic Characteristics of Patients}


We collected 396 patients with gastric adenocarcinoma who undergone gastrectomy with D2 lymphadenectomy in the first affiliated Hospital of Chongqing Medical University (Chongqing, China) from 2014 to 2015 . There were 287 patients met the standard and completed the whole follow-up evaluation(Figure 1). Table 1 details their baseline characteristics and serological results. There were 213 males $(74.6 \%)$ and 73 females $(25.4 \%)$ in the whole cohort, with an average age of $59.2 \pm 10.7$ (median = 61 ; IQR=52-66) years, 70 cases (24.4\%) with $\mathrm{BMI}>24 \mathrm{~kg} / \mathrm{m}^{2}, 47$ cases $(16.4 \%)$ with abnormal uric acid, 79 cases $(27.5 \%)$ with abnormal blood lymphocytes, and 146 cases $(50.9 \%)$ with serum albumin $\leq 4$ $\mathrm{mg} / \mathrm{dl}$. Postoperative pathological examination showed that $\mathrm{T}_{4}$ was found in 256 cases (89.2\%), and LNM existed in 209 cases (72.8\%). Postoperative pathology revealed poorly differentiated cancer in 223 cases (77.7\%), hypertension in 50 cases (17.4\%), and type 2 diabetes in 19 cases $(6.6 \%)$. In the whole cohort, the 5-year OS and DFS rates were 122/287(42.5\%) and 104/287(36.2\%), respectively. A total of 25 patients had Clavien-Dindo $\geq \otimes$ complications during postoperative hospitalization; the incidence was $8.7 \%$.

\subsection{Preoperative ULR and Clinicopathological Characteristics}

The average ULR in the whole cohort was $3.84 \pm 1.84$ (IQR=2.73-4.39). We determined the optimal cutoff value (ULR=2.95) on the ROC curve analysis. Then, 193 patients were stratified to the high-level ULR group (ULR >2.95; 67.2\%), and 94 cases were stratified to the low-level ULR group (ULR $\leq 2.95 ; 32.8 \%$ ) based on the ULR level.

We compared the clinicopathologic characteristics of the high level ULR group and low level ULR group patients. We found that age $(P<0.01)$ and gender $(P=0.04)$ were closely correlated with the ULR level. The elderly $(80.5 \%)$ and male (70.6\%) patients were mostly in the high level ULR group.

\subsection{Survival outcome in the entire cohort}

In the entire cohort, the average OS of the high level ULR group and the low level ULR group was $38.3 \pm$ 19.0 months and $46.7 \pm 18.3$ months, respectively. The 5 -year OS rates were $67 / 193(34.7 \%)$ and $55 / 94(58.5 \%)$ in the two groups. The average DFS was $36.1 \pm 18.9$ months and $44.5 \pm 19.2$ months, respectively, and the 5-year DFS rates were 55/193(28.4\%) and 45/94(52.1\%) in the two groups.

The entire cohort survival analysis showed the OS (log-rank $P<0.05)$ and DFS (log-rank $P<0.05)$ in the high level ULR group were significantly shorter than in the low level ULR group (Figure 2a-b), suggesting that a high ULR was closely related to worse OS and DFS for AGC patients.

\subsection{Univariate and Multivariate Analysis of Perioperative Parameters for Survival Outcome}

\subsection{Overall survival}

Univariate analysis suggested that a ULR $>2.95$, an abnormal peripheral blood lymphocyte count, poor differentiation, postoperative pathological $\mathrm{T}_{4}$ stage, and LNM were significantly associated with poor overall survival. Other clinicopathological variables had no statistical difference (Table 2). 
Multivariate analysis further proved that ULR $>2.95(P<0.001)$, poor differentiation $(P=0.004)$, postoperative pathological $T_{4}$ stage $(P=0.02)$, and LNM $(P<0.001)$ were independent predictors of poor OS in AGC patients (Table 2).

\subsection{Disease-free survival}

Univariate analysis showed that a ULR $>2.95$, abnormal peripheral blood lymphocyte count, poor differentiation, $T_{4}$ stage, and LNM were significantly associated with a poor prognosis of DFS in the whole cohort. The other clinicopathological variables had no statistical difference (Table 2).

Multivariate analysis further proved that a ULR $>2.95(P<0.001)$, poor differentiation $(P=0.001), T_{4}$ stage $(P=0.007)$, and LNM $(P<0.001)$ were independent predictors of poor DFS in AGC patients (Table 2).

\subsection{Propensity score matched Cohort}

To reduce the confusion bias of other covariables, we used PSM to balance the differences between the high level ULR group and the low level ULR group. SUA, blood lymphocyte count, and serum albumin were not involved in the PS balance. Based on PSM analysis, 92 pairs of well-matched data were formed, and the other covariables were fully balanced between the two groups (Table 1).

In the PSM cohort, the average OS of the high level ULR group was $36.8 \pm 19.8$ months, while the low level ULR group was $46.6 \pm 18.5$ months. The 5 -year OS rates in the high level ULR group and low level ULR group were $32.6 \%$ and $58.7 \%$, respectively.

The average DFS of patients in the PSM cohort with a high ULR was $34.9 \pm 19.8$ months, while patients with a low ULR was $44.8 \pm 19.6$ months. The 5 -year DFS rates in the high level ULR group and low level ULR group were $28.3 \%$ and $55.4 \%$, respectively.

In the PSM cohort, the survival curve showed that the OS (log-rank $\mathrm{P}<0.001)$ and DFS (log-rank $\mathrm{P}<$ 0.001) in the high level ULR group were significantly shorter than in the low level ULR group (Figure 2c-d). The above results were consistent between the PSM and entire cohorts, which further confirmed that high ULR indicated worse OS and DFS in patients with AGC who were underwent gastrectomy with D2 lymphadenectomy.

\subsection{Predictive Value of Serological Biomarkers}

ROC curve analysis were used to determine the predictive value of serological biomarkers such as ULR, SUA, Lymphocyte and Albumin for OS and DFS. In the ROC analysis based on the entire cohort of patients, ULR predicts that the AUC of OS is $0.62(95 \% \mathrm{Cl}=0.56-0.68)$, And ULR predicts that the AUC of DFS is $0.61(95 \% \mathrm{Cl}=0.55-0.68)$. Compared with the SUA, Lymphocyte and Albumin, ULR possessed the largest AUC for OS and DFS (Figure 3a-b). Further conducted ROC curve analysis based on PSM cohort, ULR predicts that the Area under the curve (AUC) of OS is $0.64(95 \% \mathrm{Cl}=0.56-0.72)$, And ULR predicts that 
the AUC of DFS is $0.63(95 \% \mathrm{Cl}=0.55-0.71)$. Similarly, ULR maintained the maximized AUC regarding to both OS and DFS of PSM cohort compared SUA, Lymphocyte and Albumin(Figure 3c-d).

\section{Discussion}

The morbidity and mortality of gastric cancer are at the forefront of all tumors, and there is a gradually increasing trend. At present, the prognostic value of some immunological and histological biomarkers for gastric cancer has been proven. However, because of the high price and complex operation, and that some markers need to be detected in the resected tumor tissue, the immunological and histological biomarkers cannot be widely used in clinical work for the time being. Therefore, it is still important to find reliable, economical, and easily available prognostic biomarkers. In this study, we assessed the prognostic value of ULR in AGC patients who had undergone gastrectomy with D2 lymphadenectomy, and we found that high levels of ULR predicted shorter OS and DFS.

Xanthine oxidoreductase (XOR) converts hypoxanthine to xanthine and then xanthine to UA. SUA is widely regarded as an effective marker for the diagnosis of gout, cardiovascular diseases, and other diseases. Although the antioxidant effect of UA was initially thought to be a protective factor for cancer, large amounts of research have realized that SUA levels are consistent with cancer mortality ${ }^{[15]}$. A large prospective study in Europe proposed that high SUA in women is independently associated with increasing overall cancer mortality ${ }^{[16]}$. A meta-analysis pointed out that humans with high SUA levels have a higher incidence of and mortality rate from cancer ${ }^{[17]}$. Previous evidence proved that SUA is not a protective factor but a risk factor for cancer. People, thus, began to explore the mechanism of the effect of uric acid on cancer. Studies have found that high SUA is associated with low adiponectin and high leptin ${ }^{[6]}$. Adiponectin may play its role on breast tumor cells partly by inhibiting Wnt signaling, Akt activity, and tumor inhibitor LKB1 ${ }^{[18][19]}$. Leptin has a tumor-promoting effect on breast, colon, prostate, and ovarian cancer in patients with obesity, metabolic syndrome, and type 2 diabetes $^{[20]}$. Studies have found that physiological levels of UA can inhibit the activity of XOR ${ }^{[21]}$, and that decreased XOR activity is associated with poor prognosis in breast, gastric, colorectal, ovarian, and lung cancer. Low XOR activity is also associated with poor differentiation and increased risk of distant metastasis in breast cancer ${ }^{[22]}$, gastric cancer ${ }^{[23]}$, and colorectal cancer ${ }^{[24]}$. In hyperuricemia, XOR activity in tumor cells is decreased. It also affects the circulating levels of adiponectin and leptin. These factors partly explain the predictive effect of high uric acid on the poor prognosis of tumors.

Peripheral blood lymphocytes are composed mainly of T cells, B cells, and a small number of NK cells. Lymphocyte reaction is the main factor that inhibits the progression of cancer ${ }^{[25]}$ especially tumorinfiltrating lymphocytes, in which CD4+ and CD8+T lymphocytes play an important role in enhancing antitumor immune response. CD4+T lymphocytes can initiate and maintain an anti-tumor immune response by secreting cytokines or activating antigen-presenting cells. CD8+T lymphocytes can recognize tumorassociated antigens and directly target tumor cells to inhibit their proliferation ${ }^{[26]}$. There has been a significant correlation between CD8+ tumor-infiltrating lymphocytes and peripheral blood lymphocyte 
count in breast cancer, with a high peripheral blood lymphocyte count predicting longer DFS in breast cancer ${ }^{[27]}$. A high peripheral blood lymphocyte count has also been significantly correlated with a good prognosis in urinary cancer ${ }^{[28]}$ and colorectal cancer ${ }^{[29]}$. A low preoperative blood lymphocyte count has been significantly associated with a poor prognosis in gastric cancer ${ }^{[30]}$.

Therefore, we combined serum uric acid with blood lymphocyte counts to reflect the combined effect of serum uric acid and lymphocyte count in the form of ULR. Recently, the predictive effect of ULR on tumor prognosis has been reported in stage I-II non-small cell lung cancer ${ }^{[15]}$. In our research, we found that the optimal cut-off point was ULR $=2.95$. All patients were stratified into a high level ULR group (ULR > 2.95) or a low level ULR group (ULR $\leq 2.95)$. The survival in the high level ULR group was significantly shorter than in the low level ULR group $(P<0.001)$. COX regression analysis showed that a ULR $>2.95$, poor differentiation, postoperative pathological $T_{4}$ stage, and LNM were independent risk factors of OS and DFS in patients with AGC after gastrectomy with D2 lymphadenectomy. We found that the older and male patients were mostly in the high level ULR group. The possible reason is that SUA in males is generally higher than that in females, and with the age increasing, the lymphocyte count decreased due to malnutrition or decreased function of bone marrow and thymus ${ }^{[30]}$. Then, to reduce the potential confounding deviation in the high and low ULR groups, PSM analysis was used. The clinicopathological features of the two groups of ULR were matched by the PS, and, finally, 92 well-matched pairs were produced. Through the analysis of the PSM cohort, ULR still had a good predictive value for OS and DFS. Moreover, ROC curve analysis showed that ULR held the best predictive value of OS and DFS compared with SUA, Lymphocyte and Albumin. Taken together, our results strongly supported that poor differentiation, postoperative pathological $\mathrm{T}_{4}$ stage, existence of LNM and ULR $>2.95$ could be regarded as independent risk factors for patients with AGC underwent gastrectomy with D2 lymphadenectomy, which meant shorter DFS and OS.

\section{Limitations}

As a single center retrospective study, due to the long follow-up time, some patients were lost to follow-up and had incomplete clinical information, which reduced the sample size and increased the bias. Finally, it should be noted that all the results of this study were obtained from a single institutional database and a small sample size. The results need to be further confirmed in the future with prospective studies and more centers, larger sample sizes, and strictly controlled variables. The specific mechanism also needs to be explored with more in vivo and in vitro experiments.

\section{Conclusion}

ULR level could independently predict the prognosis of patients with advanced gastric cancer underwent gastrectomy with D2 lymphadenectomy, with a high level of ULR indicating shorter DFS and OS.

\section{Abbreviations}




\begin{tabular}{|llll|}
\hline advanced gastric cancer & AGC & body mass index & BMI \\
\hline overall survival & OS & lymph node metastasis & LNM \\
\hline disease-free survival & DFS & propensity score matching & PSM \\
\hline uric acid & UA & area under the curve & AUC \\
\hline serum uric acid & SUA & type 2 diabetes & T2DM \\
\hline uric acid to lymphocyte ratio & ULR & Xanthine oxidoreductase & XOR \\
\hline
\end{tabular}

\section{Declarations}

\section{Ethics approval and consent to participate}

This retrospective study was approved by the Ethics Committee of Chongqing

Medical University (ID:2020-432; Chongqing, China).

\section{Consent for publication}

All authors have agreed with submission toWORLD J SURG ONCOL.

\section{Availability of data and materials}

The datasets used and/or analyzed are available from the corresponding author on reasonable request.

\section{Competing interests}

The authors declare that they have no competing interests.

\section{Funding}

Not applicable.

\section{Authors' contributions}

Li-heng Liu, Shan-shan Hu and Chenglei Liao carried out the studies, participated in collecting the data, performed the statistical analysis, and drafted the manuscript. Zi-wei Wang participated in the acquisition, analysis, and interpretation of the data. All authors read and approved the final manuscript.

\section{Acknowledgements}

Not applicable.

Reference 


\section{References}

1 Bray Freddie,Ferlay Jacques,Soerjomataram Isabelle et al. Global cancer statistics 2018: GLOBOCAN estimates of incidence and mortality worldwide for 36 cancers in 185 countries.[J] .CA Cancer J Clin, 2018, 68: 394-424.

2 Hwang Gi Yong,Baek Dong Won,Cho Hee Jeong et al. Elevated neutrophil-to-lymphocyte ratio predicts survival in patients with advanced gastric cancer treated with trastuzumab combination chemotherapy.[J] Anticancer Res., 2018, 38: 3151-3156.

3 Suzuki Takashi,Shimada Hideaki,Nanami Tatsuki et al. Hyperfibrinogenemia is associated with inflammatory mediators and poor prognosis in patients with gastric cancer.[J] Surg. Today, 2016, 46: 1394-1401.

4 Kim Do-Kyong,Oh Sung Yong,Kwon Hyuk-Chan et al. Clinical significances of preoperative serum interleukin-6 and C-reactive protein level in operable gastric cancer.[J] BMC Cancer, 2009, 9: 155.

5 Fini Mehdi A,Elias Anthony,Johnson Richard $\mathrm{J}$ et al. Contribution of uric acid to cancer risk, recurrence, and mortality.[J] Clin Transl Med, 2012, 1: 16.

6 Yang Shanshan,He Xinjia,Liu Ying et al. Prognostic significance of serum uric acid and gammaglutamyltransferase in patients with advanced gastric cancer.[J] .Dis. Markers, 2019, 2019: 1415421.

7 Chen You-Fang,Li Qiang,Chen Dong-Tai et al. Prognostic value of pre-operative serum uric acid levels in esophageal squamous cell carcinoma patients who undergo R0 esophagectomy.[J] Cancer Biomark, 2016, 17: 89-96.

8 Dziaman Tomasz,Banaszkiewicz Zbigniew,Roszkowski Krzysztof et al. 8-0xo-7,8-dihydroguanine and uric acid as efficient predictors of survival in colon cancer patients.[J] Int. J. Cancer, 2014, 134: 376-83.

9 Stotz Michael,Szkandera Joanna,Seidel Julia et al. Evaluation of uric acid as a prognostic blood-based marker in a large cohort of pancreatic cancer patients.[J] PLoS ONE, 2014, 9: e104730.

10 Kawaida Hiromichi,Kono Hiroshi,Amemiya Hidetake et al. Stratification of prognosis in patients with ampullary carcinoma after surgery by preoperative platelet-to-lymphocyte ratio and conventional tumor markers.[J] Anticancer Res., 2019, 39: 6923-6929.

11 Dirican Nigar,Karakaya Yeliz Arman,Gunes Sedat et al. Association of intra-tumoral tumour-infiltrating lymphocytes and neutrophil-to-lymphocyte ratio is an independent prognostic factor in non-small cell lung cancer.[J] Clin Respir J, 2017, 11: 789-796.

12 Wei XB, Chen WJ, Duan CY, et al. Joint effects of uric acid and lymphocyte count on adverse outcomes in elderly patients with rheumatic heart disease undergoing valve replacement surgery. $\mathrm{J}$ Thorac Cardiovasc Surg. 2019;158(2):420-427.e1. 
13 Yang Zhang,Li Shuangjiang,Zhao Liang et al. Serum uric acid to lymphocyte ratio: A novel prognostic biomarker for surgically resected early-stage lung cancer. A propensity score matching analysis.[J] Clin. Chim. Acta, 2020, 503: 35-44.

14 Li S, Yang Z, Du H, Zhang W, Che G, Liu L. Novel systemic inflammation response index to predict prognosis after thoracoscopic lung cancer surgery: a propensity score-matching study. ANZ J Surg. 2019;89(11):E507-E513.

15 Ames BN, Cathcart R, Schwiers E, Hochstein P. Uric acid provides an antioxidant defense in humans against oxidant- and radical-caused aging and cancer: a hypothesis. Proc Natl Acad Sci U S A. 1981;78(11):6858-6862. doi:10.1073/pnas.78.11.6858

16 Strasak AM, Rapp K, Hilbe W, et al. The role of serum uric acid as an antioxidant protecting against cancer: prospective study in more than 28000 older Austrian women. Ann Oncol. 2007;18(11):1893-1897. doi:10.1093/annonc/mdm338

17 Yan S, Zhang P, Xu W, et al. Serum uric acid increases risk of cancer incidence and mortality: A systematic review and meta-analysis. Mediators Inflamm. 2015;2015:764250. doi:10.1155/2015/764250

18 Ames BN, Cathcart R, Schwiers E, Hochstein P. Uric acid provides an antioxidant defense in humans against oxidant- and radical-caused aging and cancer: a hypothesis. Proc Natl Acad Sci U S A. 1981;78(11):6858-6862. doi:10.1073/pnas.78.11.6858

19 Taliaferro-Smith L, Nagalingam A, Zhong D, Zhou W, Saxena NK, Sharma D. LKB1 is required for adiponectin-mediated modulation of AMPK-S6K axis and inhibition of migration and invasion of breast cancer cells. Oncogene. 2009;28(29):2621-2633. doi:10.1038/onc.2009.129

20 Khandekar MJ, Cohen P, Spiegelman BM. Molecular mechanisms of cancer development in obesity. Nat Rev Cancer. 2011;11(12):886-895. Published 2011

21 Tan S, Radi R, Gaudier F, et al. Physiologic levels of uric acid inhibit xanthine oxidase in human plasma. Pediatr Res. 1993;34(3):303-307.

22 Linder N, Lundin J, Isola J, Lundin M, Raivio KO, Joensuu H. Down-regulated xanthine oxidoreductase is a feature of aggressive breast cancer. Clin Cancer Res. 2005;11(12):4372-4381. doi:10.1158/10780432.CCR-04-2280

23 Linder N, Haglund C, Lundin M, et al. Decreased xanthine oxidoreductase is a predictor of poor prognosis in early-stage gastric cancer. J Clin Pathol. 2006;59(9):965-971. doi:10.1136/jcp.2005.032524

24 Linder N, Martelin E, Lundin M, et al. Xanthine oxidoreductase - clinical significance in colorectal cancer and in vitro expression of the protein in human colon cancer cells. Eur J Cancer. 2009;45(4):648655. doi:10.1016/j.ejca.2008.10.036 
25 Dunn Gavin P,Old Lloyd J,Schreiber Robert D, The immunobiology of cancer immunosurveillance and immunoediting.[J] Immunity, 2004, 21: 137-48.

26 Ino Y,Yamazaki-ltoh R,Shimada $\mathrm{K}$ et al. Immune cell infiltration as an indicator of the immune microenvironment of pancreatic cancer.[J] Br. J. Cancer, 2013, 108: 914-23.

27 Lee KH, Kim EY, Yun JS, et al. The prognostic and predictive value of tumor-infiltrating lymphocytes and hematologic parameters in patients with breast cancer. BMC Cancer. 2018;18(1):938. Published 2018 Oct 1.

28 Yakovlev P, Klyushin D. Lymphocyte count in peripheral blood is a sensitive tool in pretreatment assessment of patients with urological cancer. Exp Oncol. 2018;40(2):119-123.

29 Iseki Y, Shibutani M, Maeda K, et al. The impact of the preoperative peripheral lymphocyte count and lymphocyte percentage in patients with colorectal cancer. Surg Today. 2017;47(6):743-754.

30 Tatara Takashi,Suzuki Satoshi,Kanaji Shingo et al. Lymphopenia predicts poor prognosis in older gastric cancer patients after curative gastrectomy.[J] Geriatr Gerontol Int, 2019, 19: 1215-1219.

\section{Tables}

Due to technical limitations, table 1 and 2 is only available as a download in the Supplemental Files section.

\section{Figures}




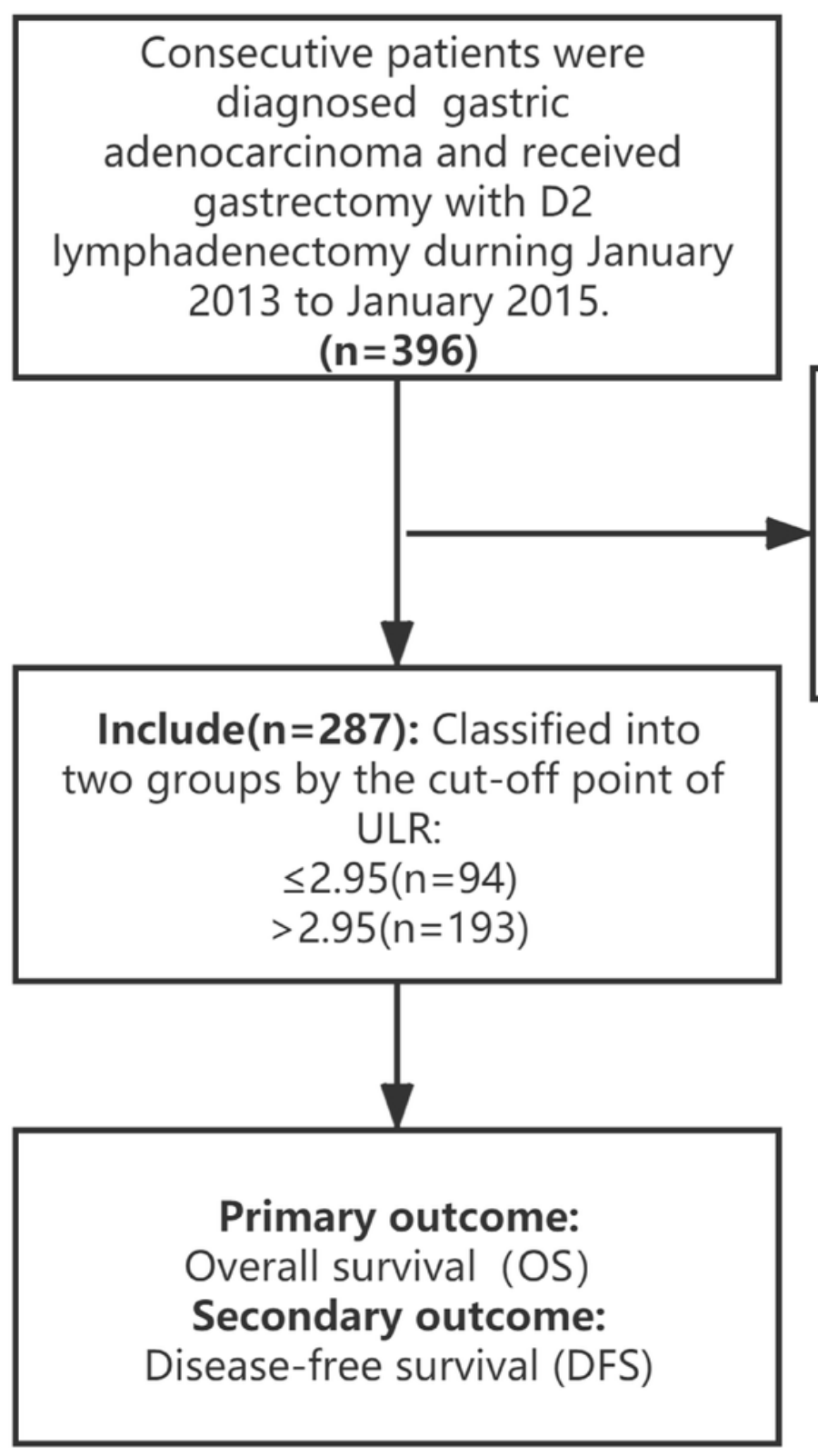

\section{Exclude $(n=109)$ : \\ T1 stage $(n=28)$}

Loss of follow-up $(n=43)$

Received preoperative therapy $(n=15)$ Previous diseases affecting serum uric acid and blood lymphocyte $(n=23)$

\section{Figure 1}

Patient selection of study cohort. ULR, Uric acid to lymphocyte count ratio. 

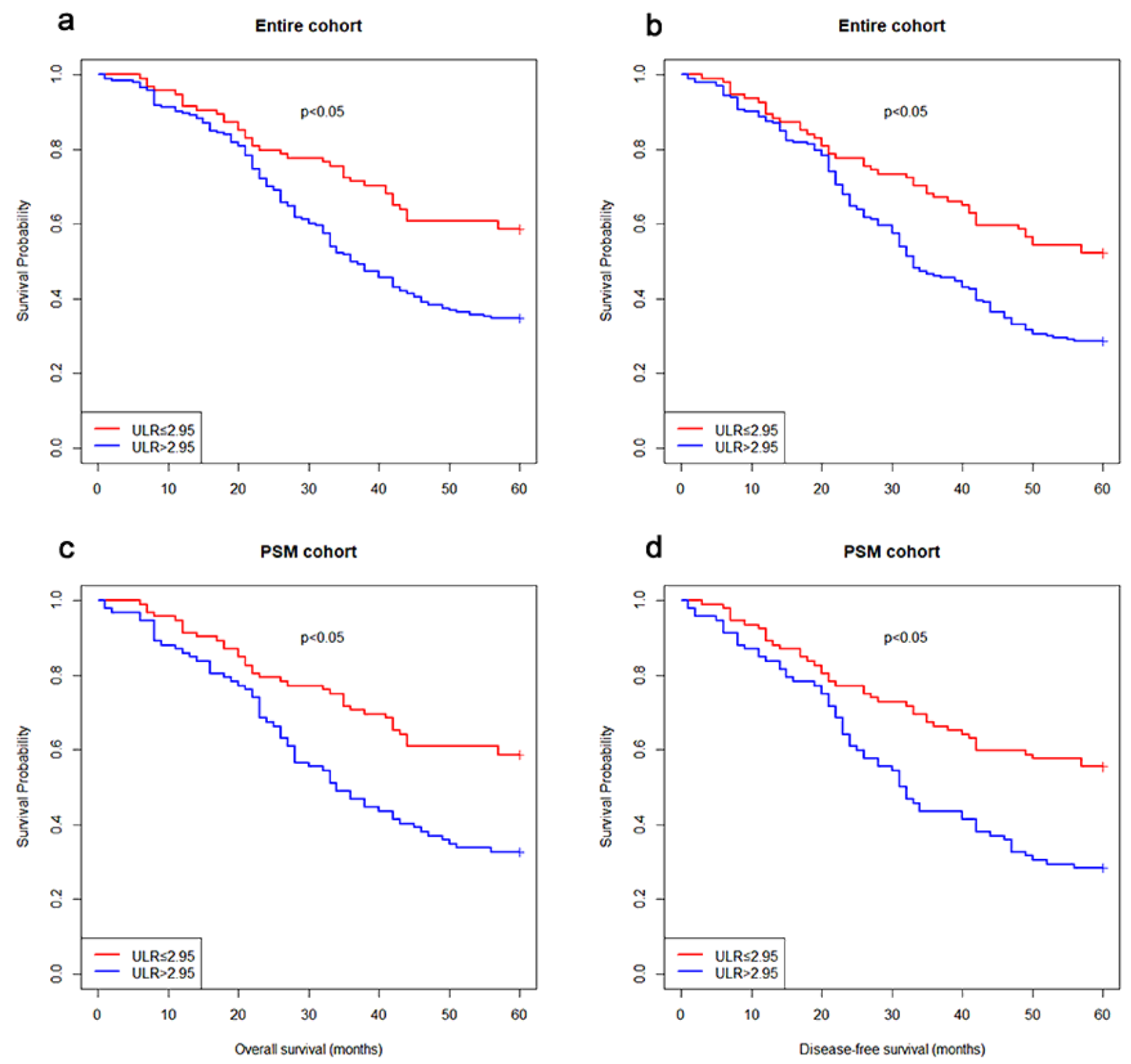

\section{Figure 2}

Kaplan-Meier curves for OS and DFS in two cohort. Notes: (a) OS curves of entire cohort in the low-ULR group( $(\leq 2.95)$ vs the high-ULR group (>2.95), $p<0.05$; (b) DFS curves of entire cohort in the low-ULR group $(\leq 2.95)$ vs the high-ULR group (>2.95), $p<0.05$; (c)OS curves of PSM cohort in the low-ULR group ( $\leq 2.95$ ) vs the high-ULR group ( $>2.95), p<0.05$;(d)DFS curves of PSM cohort in the low-ULR group $(\leq 2.95)$ vs the high-ULR group (>2.95), $p<0.05$; 

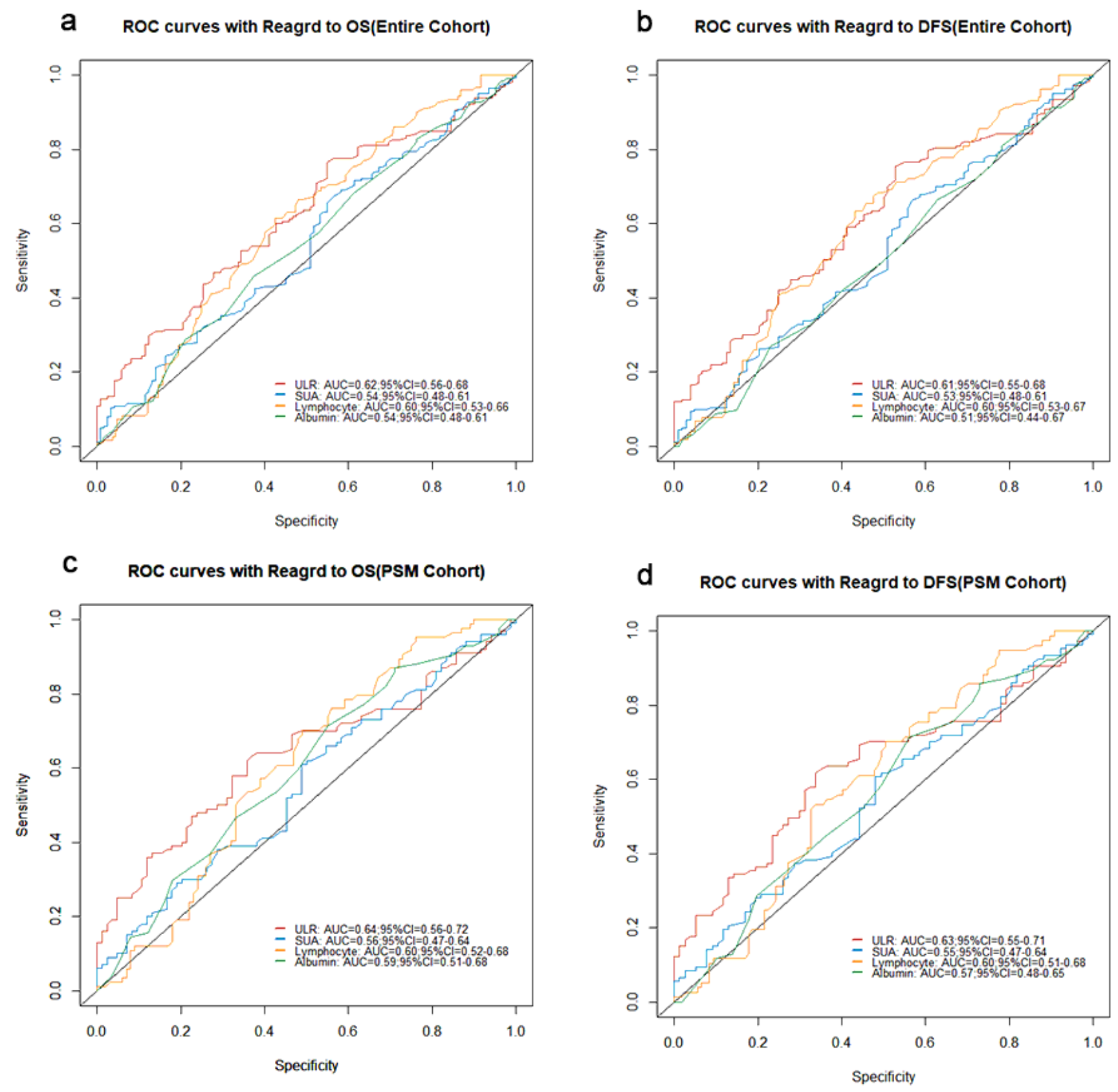

\section{Figure 3}

ROC curves of ULR, uric acid, lymphocyte count, and Albumin for OS and DFS.Notes:(a)ROC curves of serological biomarkers for OS of entire cohort.(b)ROC curves of serological biomarkers for DFS of entire cohort.(c)ROC curves of serological biomarkers for OS of PSM cohort.(d)ROC curves of serological biomarkers for DFS of PSM cohort. AUC, Area under the curve; $\mathrm{Cl}$, confidence interval; ULR, uric acid to lymphocyte count ratio. 


\section{Supplementary Files}

This is a list of supplementary files associated with this preprint. Click to download.

- Table1.pdf

- Table2.pdf 